\title{
Hepatic multiple perivascular epithelioid cell neoplasm: A case report and literature review
}

\author{
YIN ZHI LAN and XIAO EN HUA \\ The Second Xiangya Hospital of Central South University, Changsha, Hunan 410011, P.R. China
}

Received October 5, 2015; Accepted January 5, 2016

DOI: $10.3892 / \mathrm{mco} .2016 .735$

\begin{abstract}
Perivascular epithelioid cell neoplasm (PEComa) is a rare tumor type and primary hepatic multiple perivascular is rarer still. The present case report investigated the case of a 40-year-old woman who was admitted to The Second Xiangya Hospital for hepatic multiple tumor by physical examination without any history of abdominal pain or body weight loss. Abdominal plain computed tomography revealed three lesions in the liver. All lesions exhibited a well-defined boarder and only one mass contained fatty tissue in S6 of the liver. An enhanced scan revealed that all lesions were heterogeneous, and were enhanced on the artery phase and on portal vein phase. On delay phase, the lesions revealed continued enhancement, which is isoattenuating to the normal hepatic parenchyma. Following excision of the three masses, the patient was pathologically diagnosed with hepatic multiple PEComa. Short-term re-examination revealed no recurrence.
\end{abstract}

\section{Introduction}

Perivascular epithelioid cell neoplasm (PEComa) is a rare tumor type (1) and was first identified in 1992. PEComa was defined as a mesenchymal tumor composed of histologically and immunohistochemically distinctive perivascular epithelioid cells by the World Health Organization in 2002. It is a rare tumor type, comprising a group of mesenchymal neoplasms, including angiomyolipoma (AML), clear cell 'sugar' tumor (CCST) of the lung, lymphangioleiomyomatosis (LAM), and a variety of unusual visceral, intra-abdominal and soft tissue/bone tumors. PEComa rarely originate in the liver, particularly multiple PEComas (2). To the best of our knowledge, few cases of hepatic multiple PEComas have been described in the literature to date (3-5). Due to the small number of reported cases, the imaging and prognosis of the tumor remain to be adequately determined. The present study

Correspondence to: Miss Yin Zhi Lan, The Second Xiangya Hospital of Central South University, 139 Middle Renmin Road, Changsha, Hunan 410011, P.R. China

E-mail: longyangli@126.com

Key words: perivascular epithelioid cell neoplasm, liver, multifocal described the case of a 40-year-old female patient with hepatic multiple PEComa.

\section{Case report}

A 40-year-old female was admitted to The Second Xiangya Hospital for hepatic multiple tumor by physical examination without any history of abdominal pain or body weight loss. During laboratory examination, no abnormalities were identified in serum $\alpha$-fetoprotein or by standard blood tests and chest X-rays. Abdominal plain computed tomography (CT) revealed three lesions (Fig. 1) in S4, S5 and S6 of the liver. The tumor sizes were $9.4 \times 6.5 \times 5.3,4.5 \times 5 \times 3.1$ and $2.2 \times 2.5 \times 2.3 \mathrm{~cm}$, respectively. All lesions exhibited a well-defined boarder and only one mass contained fatty tissue in S6 of the liver. An enhanced scan on arterial phase images revealed that all lesions were avidly heterogeneous and enhanced, with the exception of fatty tissue. The lesions exhibit hyperattenuating or isoattenuating enhancement relative to adjacent hepatic parenchyma in the portal vein and delay phase. During surgery, all lesions were resected. The cut surface was described as soft and areas of hemorrhage were present in the largest lesions. Immunohistochemistry revealed that the tumor cells were markedly positive for human melanoma black (HMB)-45 and Ki-67 (+, 2\%), whereas the tumor was negative for S-100 protein. The patient recovered well post-operation and no recurrence was identified during the follow-up the following year. The present study was approved by the Ethics Committee of the Second Xiangya Hospital of Central South University (Changsha, China), and written informed consent was obtained from the patient.

\section{Discussion}

PEComa is a rare tumor type, which originated in mesenchymal tissues. PEComa (1) was first identified in 1992 and was defined in 2002 as a mesenchymal tumor composed of histologically and immunohistochemically distinctive perivascular epithelioid cells by the World Health Organization. It is a rare tumor type, comprising a group of mesenchymal neoplasms, including AML, CCST of the lung, LAM, and a variety of unusual visceral, intra-abdominal and soft tissue/bone tumor types (2). It has been previously reported that the tumor may arise from multiple sites, including the kidney, liver, lung, retroperitoneum, abdominal wall, extremities and neck $(6,7)$. 

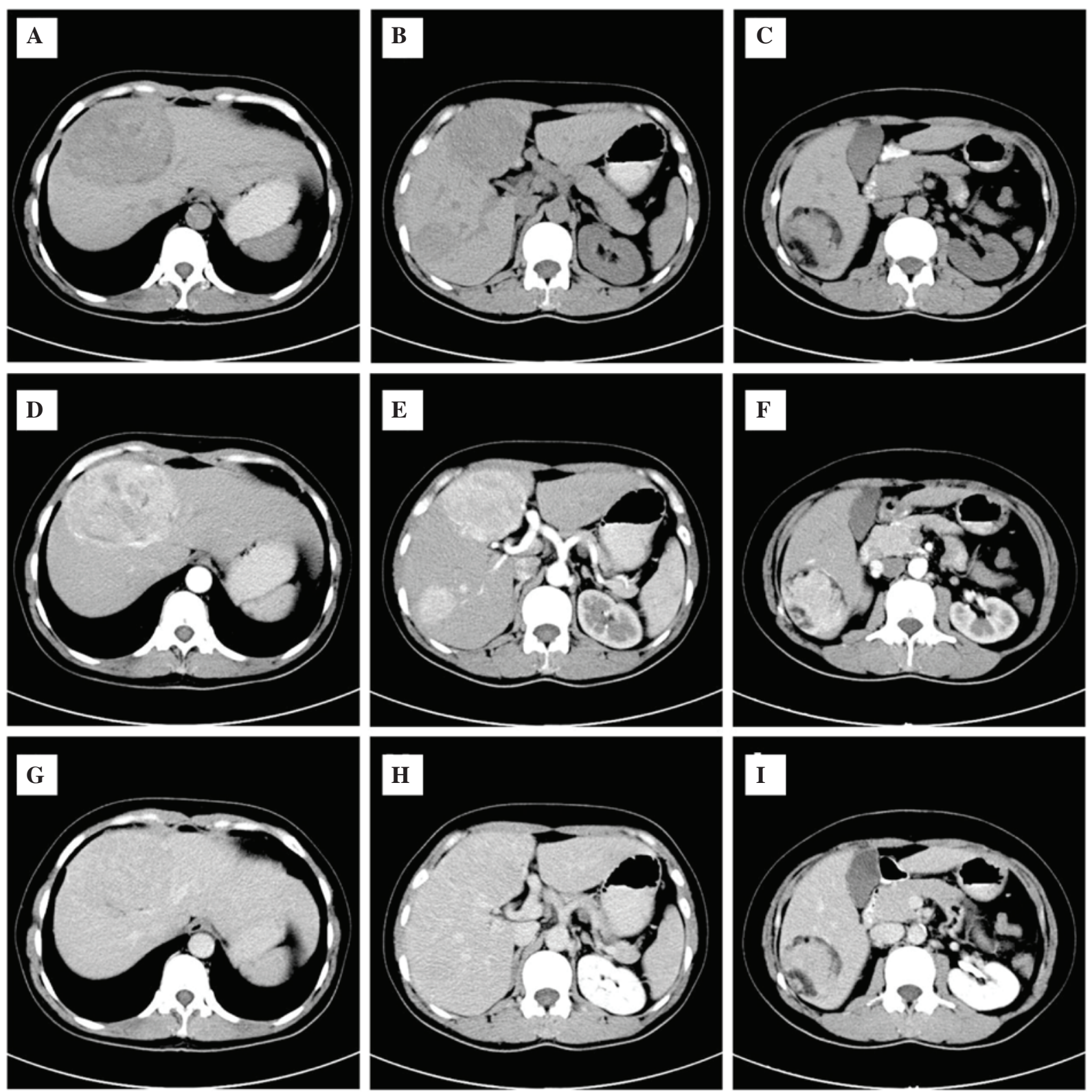

Figure 1. A CT image of hepatic multiple perivascular epithelioid cell neoplasm. (A-C) The plain CT scan revealed three well-demarcated masses with heterogeneous density in the liver and one mass in the S6 contained macroscopic fat. (D-F) Contrast-enhanced CT scan of the masses revealed heterogeneously enhanced lesions in the arterial phase on and (G-I) partial phase image lesions revealed that the lesion had returned to an isoattenuating state. CT, computed tomography.

The majority of hepatic lesions are a single focus, usually accompanying patients with tuberous sclerosis. Although PEComas exhibit a wide spectrum of biological behavior, experts classified it into three types: i) Benign; ii) of uncertain malignant potential; iii) malignant $(8,9)$. The imaging features of malignant PEComas include signs of tumor size $>5 \mathrm{~cm}$, infiltrative growth pattern, high nuclear grade and hypercellularity, a high rate of mitosis, $>1 / 50$ high-power fields, coagulative necrosis and vascular invasion $(10,11)$. The histopathological criteria for the diagnosis of malignant PEComa remains to be established, as a result of its rarity in literature. Notably, the above criteria remain to be validated in a larger series.

PEComas coexpress melanocytic markers, including gp100 protein (HMB-45), Melan-A, tyrosinase and MiTF, and muscle markers, including SMA, pan-muscle actin, muscle myosin, calponin and occasionally h-caldesmon, with desmin and cytokeratin (12-15). The most sensitive markers are desmin and HMB-45, followed by SMA and caldesmon (8). In the present case, HMB-45 and cytokeratin were positively expressed, whereas the present samples were negative for S-100 protein.

This tumor type is more predominant in female individuals, typically middle-aged patients. Imaging studies can confirm the presence of the tumor, its location, size, inner echostructure and association with other organs. Diagnostic imaging of PEComa shows a wide variety of patterns. PEComas are associated with specific imaging of detention of fat components on CT or magnetic resonance imaging (MRI). Solitary tumors were more common compared with multiple masses. The majority of tumors were large in size, particularly 
in retroperitoneal PEComas, have a well-defined border and are of regular shape. Certain lesions were isodense with fat. The enhancement CT or MRI following contrast agent administration is usually enhanced heterogeneously. This can be significantly enhancement on arterial and venous phases. The tumors appeared slightly hypodense or isodense on delayed CT or MRI (11,16-18). No clear distinction was observed between the benign and malignant counterparts in the majority of the radiology case reports, case series and reviews. With a size $>5 \mathrm{~cm}$, however absent further risk factors, including infiltrative growth pattern, high nuclear grade and cellularity, mitotic rate $>1 / 50$ high-power fields, necrosis or vascular invasion, and stratified as 'uncertain malignant potential', according to the modified Folpe criteria (19).

As a result of an extensive range of biological behaviors, the treatment for PEComas has been a difficult issue to conclude (20). Although numerous determining factors, including tumor size, growth pattern, necrosis and nuclear grade, indicated a degree for malignancy, most PEComas appear to be benign. The majority are treated with surgery alone (21). In fact, adjuvant therapy is recommended for all patients with malignant features or metastasis (22). The current patient received surgery alone. Three masses were completely excised and no further treatment was applied. No recurrence was observed during the follow-up at 1 year post-surgery.

In conclusion, hepatic multiple PEComa is a rare tumor type. If the mass contains fat, the diagnosis is relatively simple. Whether hepatic multiple PEComa or hepatic isolate PEComa have a difference prognostic remains uncertain, however, should be further evaluated.

\section{References}

1. Bonetti F, Pea M, Martignoni G and Zamboni G: PEC and Sugar. Am J Surg Pathol 16: 307-308, 1992.

2. Folpe AL and Kwiatkowski DJ: Perivascular epithelioid cell neoplasms: Pathology and pathogenesis. Hum Pathol 41: 1-15, 2010.

3. Anysz-Grodzicka A, Pacho R, Grodzicki M, Koperski L, Górnicka B, Cieszanowski A, Zieniewicz K and Krawczyk M: Angiomyolipoma of the liver: Analysis of typical features and pitfalls based on own experience and literature. Clini Imaging 37: 320-326, 2013

4. Kumasaka S, Arisaka Y, Tokue A, Higuchi T, Nakajima T and Tsushima Y: A case of multiple hepatic angiomyolipomas with high (18) F-fluorodeoxyglucose uptake. BMC Med Imaging 14: 17, 2014.

5. Nonomura A, Mizukami Y, Kadoya M, Takayanagi N and Hirono T: Multiple angiomyolipoma of the liver. J Clin Gastroenterol 20: 248-251, 1995.
6. Desy NM, Bernstein M, Nahal A, Aziz M, Kenan S, Turcotte RE and Kahn LB: Primary perivascular epithelioid cell neoplasm (PEComa) of bone: Report of two cases and review of the literature. Skeletal Radiol 41: 1469-1474, 2012.

7. Armah HB and Parwani AV: Perivascular epithelioid cell tumor. Arch Pathol Lab Med 133: 648-654, 2009.

8. Thway K and Fisher C: PEComa: Morphology and genetics of a complex tumor family. Ann Diagn Pathol 19: 359-368, 2015.

9. Fu X, Jiang JH, Gu X and Li Z: Malignant perivascular epithelioid cell tumor of mesentery with lymph node involvement: A case report and review of literature. Diagn Pathol 8: 60, 2013.

10. Yamamoto H, Oda Y, Yao T, Oiwa T, Kobayashi C, Tamiya S, Kawaguchi K, Hino O and Tsuneyoshi M: Malignant perivascular epithelioid cell tumor of the colon: Report of a case with molecular analysis. Pathol Int 56: 46-50, 2006.

11. Tirumani SH, Shinagare AB, Hargreaves J, Jagannathan JP, Hornick JL, Wagner AJ and Ramaiya NH: Imaging features of primary and metastatic malignant perivascular epithelioid cell tumors. AJR Am J Roentgenol 202: 252-258, 2014.

12. Pea M,Bonetti F,ZamboniG, Martignoni G, Riva M,Colombari R, Mombello A, Bonzanini M, Scarpa A, Ghimenton C, et al: Melanocyte-marker-HMB-45 is regularly expressed in angiomyolipoma of the kidney. Pathology 23: 185-188, 1991.

13. Wu K and Tazelaar HD: Pulmonary angiomyolipoma and multifocal micronodular pneumocyte hyperplasia associated with tuberous sclerosis. Hum Pathol 30: 1266-1268, 1999.

14. Hornick JL and Fletcher CD: Sclerosing PEComa: Clinicopathologic analysis of a distinctive variant with a predilection for the retroperitoneum. Am J Surg Pathol 32: 493-501, 2008.

15. Stone CH, Lee MW, Amin MB, Yaziji H, Gown AM, Ro JY, Têtu B, Paraf F and Zarbo RJ: Renal angiomyolipoma: Further immunophenotypic characterization of an expanding morphologic spectrum. Arch Pathol Lab Med 125: 751-758, 2001.

16. Tan Y, Zhang H and Xiao EH: Perivascular epithelioid cell tumor: Dynamic CT, MRI and clinicopathological characteristics-analysis of 32 cases and review of the literature. Clin Radiol 68: 555-561, 2013

17. Kloth C, Boozari B, Bösmüller H, Haap M and Horger M: Multimodality imaging of hepatic PEComa. RoFo 187: 147-150, 2015.

18. Tan Y and Xiao EH: Hepatic perivascular epithelioid cell tumor (PEComa): Dynamic CT, MRI, ultrasonography and pathologic features-analysis of 7 cases and review of the literature. Abdom Imaging 37: 781-787, 2012.

19. Bleeker JS, Quevedo JF and Folpe AL: 'Malignant' perivascular epithelioid cell neoplasm: Risk stratification and treatment strategies. Sarcoma 2012: 541626, 2012.

20. Pan CC, Jong YJ, Chai CY, Huang SH and Chen YJ: Comparative genomic hybridization study of perivascular epithelioid cell tumor: Molecular genetic evidence of perivascular epithelioid cell tumor as a distinctive neoplasm. Hum Pathol 37: 606-612, 2006.

21. Fadare O: Perivascular epithelioid cell tumor (PEComa) of the uterus: An outcome-based clinicopathologic analysis of 41 reported cases. Adv Anat Pathol 15: 63-75, 2008

22. Zekry N, Rettenmaier MA, Abaid LN, John CR, Micha JP, Brown JV III and Goldstein BH: Perivascular epithelioid cell neoplasms: A systematic review of prognostic factors. J Minim Invasive Gynecol 16: 527-532, 2009. 